\title{
Identification Hub Genes of Consensus Molecular Subtype Correlation with Immune Infiltration and Predict Prognosis in Gastric Cancer
}

Bin Yu

First Affiliated Hospital of Nanchang University

Xin Yu

First Affiliated Hospital of Nanchang University

Jianping Xiong

First Affiliated Hospital of Nanchang University

Mei Ma ( $\nabla$ mamei@ncu.edu.cn )

First Affiliated Hospital of Nanchang University https://orcid.org/0000-0002-4656-3752

\section{Research Article}

Keywords: Gastric Cancer, Prognosis, Immune Infiltration, OGN, CHRDL2

Posted Date: July 12th, 2021

DOI: https://doi.org/10.21203/rs.3.rs-692503/v1

License: (c) (i) This work is licensed under a Creative Commons Attribution 4.0 International License.

Read Full License 


\title{
Identification Hub Genes of Consensus Molecular Subtype Correlation with Immune Infiltration and Predict Prognosis in Gastric Cancer
}

Bin $\mathrm{Yu}^{1}$, Xin $\mathrm{Yu}^{2}$, Jianping Xiong ${ }^{2}, \mathrm{Mei} \mathrm{Ma}^{2}$

${ }^{1}$ Department of General Surgery, The First Affiliated Hospital of Nanchang University, Nanchang, Jiangxi Province, 330006

${ }^{2}$ Department of Oncology, The First Affiliated Hospital of Nanchang University, Nanchang, Jiangxi Province, 330006

*Correspondence: mamei@ncu.edu.cn, xiong_jp_ncu@126.com

\begin{abstract}
Gastric Cancer (GC) has a great fatality rate, meanwhile, there is still a lack of available biomarkers for prognosis. The goal of the research was to discover key and novel potential biomarkers for GC. We screened for the expression of significantly altered genes based on survival rates from two consensus molecular subtypes (CMS) of GC. Subsequently, functional enrichment analysis showed these genes involved in many cancers. And we picked 6 hub genes that could both secreted in the tumor microenvironment and expression enhanced in immune cells. Then, Kaplan Meier survival and expression detected in the tumor pathological stage were utilized to clarify the prognostic of these 6 hub genes. The results indicated that OGN, CHRDL2, C2orf40, THBS4, CHRDL1, and ANGPTL1, respectively, were significantly associated with poor $\mathrm{OS}$ in GC patients. And their expression increased with cancer advanced. Moreover, immune infiltration analysis displayed that those hub genes expression positively with M2 macrophage, CD8+ T Cell, most immune inhibitors, and majority
\end{abstract}


immunostimulators. In summary, our results suggested that OGN, CHRDL2, C2orf40, THBS4, CHRDL1, and ANGPTL1 were all potential biomarkers for GC prognosis and might also be potential therapeutic targets for GC.

Keywords Gastric Cancer, Prognosis, Immune Infiltration, OGN, CHRDL2

\section{Introduction}

Gastric Cancer (GC) is one of the most common malignant tumors, and its mortality ranks fourth among all malignant tumors in the world[1]. According to the global cancer statistics (2020), there were over one million new cases of GC and with a mortality rate of $7.7 \%$ of the total cancer deaths[1]. Due to the lack of effective biomarkers, most patients with GC are diagnosed at an advanced stage and distant metastasis often occurs at this stage, leading to a poor prognosis. The 5-year overall survival rate of GC is about $20 \%-30 \%$ in most parts of the world[2]. The earlier the effective intervention for GC patients, the more beneficial to improve their survival rate. Therefore, it is necessary to identify the key prognostic biomarkers in early time for GC therapy.

Immune checkpoint blockade (ICB) has been brought new hope for many cancers treatment. While ICB does not valid in most cases, including GC[3], and thus more research is required to drive the field forward. Immune-related cells in the tumor microenvironment (TME) also indispensable for the development, progression, and prognosis of GC. Despite CD8+T cells are considered to be an anti-tumor component in the TME[4], studies on the relationship between CD8+T cells and prognosis in GC yielded in divergence[5, 6]. Programmed cell death protein 1 (PD-1) is an important immune checkpoint molecule in $\mathrm{CD} 8+\mathrm{T}$ cell and its upregulation disturbs the anti- 
tumor function of $\mathrm{CD} 8+\mathrm{T}$ cell[7]. $\mathrm{PD}-1+\mathrm{CD} 8+\mathrm{T}$ cell infiltration was significantly increased in patients with GC[6]. While PD-1 blockade combined with immune modulators, such as TGF- $\beta 1$ inhibitor, might take patients more benefits than targeted PD-1 alone [6]. And it was also reported that some subsets of $\mathrm{CD} 8+\mathrm{T}$, such as CXCR5+CD8+T[8], but not CD8+T itself connected with better overall survival (OS) in patients with GC. Therefore, it is important to find out the key molecules that regulate immune modulators in TME or/and co-express molecular in $\mathrm{CD} 8+\mathrm{T}$ that mediated its function.

Besides, the latest studies have proved that macrophages, mostly M2 polarized macrophages, have a vital role in the GC advance[9, 10]. Infiltration of M2 macrophage might be a worthwhile biomarker for GC prognosis[11]. Targeting M2 macrophages may be a promising therapeutic strategy, therefore, it is valuable that uncover M2related molecules.

Here, we picked out those genes that were significantly associated with the survival of GC patients. And selected hub genes, OGN, CHRDL2, C2orf40, THBS4, CHRDL1, and ANGPTL1, which secreted into TME and enhanced in immune cells. After validated their expression value for GC diagnosis, we performed immune infiltration analysis of these 6 hub genes. Our study might provide novel biomarkers for GC advance, and potential targets that combined using with ICB to improve GC therapy.

\section{Results}

\section{Identification of robust genes associated with GC survival}

The COMSUC web server[12] was used to find out the hub genes significantly 
correlated with the survival of GC patients. we firstly clustered GC samples into two consensus molecular subtypes (CMS) by multiple methods, including K-means, hierarchical clustering (Hclust), non-negative matrix factorization (NMF) (Figure. 1ab). And we found that CMS1 had lower survival than CMS2 (Figure. 1c). 52 genes prominently up-regulated expression in CMS1 compare to CMS2 (Figure. 1d). These genes may act independently or work in concert for GC survival.

\section{Functional enrichment analysis of survival-related CMS genes}

To identify the notably enriched pathway and biological process of survival-related CMS genes, we explored in Metascape website [13] and found those genes related to many processes of cancer (Figure. 2a). And the most relative pathway was early gastric cancer (Figure. 2a). Then we analyzed the sub-network of those CMS1 genes in TCGA RNAseq of STAD, most of them had some connections in each other (Figure. 2b). According to those results, we speculated whether those genes were regulated by some common transcription factors (TFs). After performing TF enrichment in Knock TF (http://www.licpathway.net/KnockTF/index.html), more than ten TFs predicted may regulate over $73 \%$ of genes of these selected 52 genes (Table 1 ). The top 4 TFs were TFAP4, TP53, CREB1, and PTEN, respectively (Figure. 2c), and they all have been reported to promote the progression of GC [14-17]. Furthermore, we explored the characteristics of the 52 genes in the GeneCards (https://www.genecards.org/) and the Human Protein Atlas (https://www.proteinatlas.org/). As shown in Table 2, 10 genes could be transcribed into secreted proteins, 22 genes enriched in immune cells and only 7 genes own both characters (Fig. 2d). 


\section{Survival outcomes in selected genes}

To verify whether the selected 7 genes (OGN, CHRDL2, C2orf40, THBS4, CHRDL1, ANGPTL1, SLIT2) were correlated with worse overall survival (OS) in GC patients, survival analysis was performed in Kaplan Meier plotter [18]. On account of many articles that have been shown that SLIT2 was over-expression in gastric cancer and might be an independent risk factor for GC $[19,20]$, we did not explore it further in this paper. The Kaplan Meier plotter results showed that the up-regulated expression of OGN, CHRDL2, C2orf40, THBS4, CHRDL1, and ANGPTL1, respectively, was correlated with poorer OS in GC patients (Fig. 3a-f). Among them, OGN and CHRDL2 were firstly reported in GC that their expression was related to OS of GC patients. To further confirm the correlation of 6 genes expression with tumor progression in GC, expression in different stages was executed in GEPIA[21]. The analyzed results indicated that the expression of OGN, CHRDL2, C2orf40, THBS4, CHRDL1, and ANGPTL1 was gradually increased in the GC stage (Fig. 4a-f). These results revealed these 6 hub genes were significantly related to the aggravation and survival of GC.

\section{Immune cell infiltration analysis}

To investigate the relationship between the expression of these hub genes (OGN, CHRDL2, C2orf40, THBS4, CHRDL1, and ANGPTL1) and immune infiltration, the TIMER website[22] was used to analyze the correlation between these hub genes and multiple immune cells. The results indicated that the expression of these hub genes was significantly positively correlated with CD8+ T Cell, CD4+ T Cell, macrophage, neutrophil, dendritic cell (Fig. 5). Next, we further analyzed the correlations of 
macrophage subtypes infiltration with these hub genes in GC. The results exhibited these 6 hub genes had no significant link in M1-related markers with their expression, but exhibited remarkable connection in M2 and TAMs (Fig. 6).

\section{Correlation analysis between hub genes and immune markers}

To better understand the immune function of these hub genes, we explored the relationship between their expression and immune markers in TISIDB[23]. Tumor immunology analysis exhibited that the expression of these 6 genes was positively correlated with most immunoinhibitors (Fig. 7a) and majority immunostimulators (Fig.

8a). Furthermore, we check out the striking different genes verified again in GEPIA, like CSF1R and Cxc112, and got similar results (Fig. 7b-g, 8b-g). In the end, we search data found that OGN, C2orf40, CHRDL2, THBS4, CHRDL1, and ANGPTL1 had different immune subtypes in GC (Fig. 9a-f) and their expression was divergent in various molecular subtypes (Fig. 10a-f). In summary, these results implied that these 6 hub genes might potentially govern the conscription and awakening of immune cells in GC.

\section{Discussion}

Chemotherapy, radiotherapy, surgery, immunotherapy, and targeted therapy are effective in the progression of GC in appropriate indications. While the limited OS that no more than $30 \%$ in most countries, there are still need specific biomarkers to guide rational using drugs, especially for ICB treatment.

OGN is a member of the small leucine-rich proteoglycan (SLRP) family and its function may vary from different tumors. It was reported that OGN was upregulated in 
meningioma, and function as a tumor promoter by inhibiting NF2 expression and triggering mTOR signaling [24]. There was, however, also experimental evidence that OGN expression reduced in multiple cancers and its expression levels positively with survival rates[25, 26]. For example, OGN inhibited cell proliferation, invasion, and epithelial to mesenchymal transition through EGFR/Akt pathway in colorectal cancers [27]. Besides, OGN, as one of the biologically active elements of the vascular extracellular matrix, could be tested in plasma/serum and acted as a biomarker in disease [28, 29]. For instance, serum OGN was an independent risk predictor for patients with chronic kidney disease [28]. OGN also could adjust immune response via mediating immune cell infiltration in cancer, such as OGN expression positively associated with CD8 $+\mathrm{T}$ cells recruitment/infiltration by inhibited HIF-1 $\alpha /$ VEGF pathway in colorectal cancer[26]. The role of OGN in GC has not been reported so far. Our study showed that OGN could be a new useful prognostic biomarker and immune regulator for GC.

CHRDL2, an antagonist of bone morphogenic proteins (BMPs), worked as an oncogene in colorectal cancer [30] and osteosarcoma [31]. CHRDL2 was firstly discovered in 2003 named as BNF-1 (breast tumor novel factor 1) and found its overexpression in breast, lung, and colon tumors detected by PCR in a small sample[32]. While the report of CHRDL2 in the tumor is very limited. Here, we first pointed out that CHRDL2 expression increased in the GC process and its high expression was associated with poor prognosis in GC patients. As a secreted protein, we found its expression associated with immune cell infiltration, especially for M2 macrophage, and immune inhibit 
molecular markers. Therefore, CHRDL2 might be a novel target of GC therapy. C2orf40 encodes a protein called esophageal cancer-related gene-4 (ECRG4), which is down-regulated by hypermethylation of its promoter in diverse types of tumors, including hepatocellular carcinoma [33], gastric cancer [34], breast cancer [35]. According to current reports, c2orf40 might as a potential tumor suppressor gene in tumors. However, our results exhibited that high ECRG4 expression was remarkably associated with poorer outcomes $(\mathrm{HR}=1.95$, log-rank $\mathrm{P}=6.7 \mathrm{e}-10)$, and its expression showing a state of gradual increase in GC advance. The opposite result may be on account of different levels of evidence or cohort studies $[34,36]$. The deep reasons yet to be further validated. It has been reported that ECRG4 interacted with TLR4 [34]. And TLR4/PI3K/Akt signaling was a vital way to promote M2 polarization of macrophages [10]. It also needs to verify whether ECRG4 is involved in the development of GC by modifying the M2 polarization of macrophages through TLR4. THBS4 belongs to the thrombospondin protein family, which is a kind of adhesive glycoproteins that mediate cell-to-cell and cell-to-matrix interactions. As a secreted extracellular matrix protein, THBS4 has been reported mediated angiogenesis responding to TGF- $\beta$ signaling [37]. THBS4 in favor of an oncogene in colorectal cancer [38], prostate cancer [39], and hepatocellular carcinoma [40]. Compared to normal tissues, THBS4 expression at a higher level in those cancers. In colorectal cancer, THBS4 secretion induced by TGF $\beta$ / PDGFR $\beta$ pathways and promoted adhesion, migration, and proliferation of colorectal cancer [38]. And THBS4 might as a potential therapeutic target in prostate cancer, inhibited expression of THBS4 could impede the 
PI3K/Akt signaling and disturb the cancer stem cell (CSC)-like properties[39]. THBS4 might as a biomarker for diffuse-type gastric adenocarcinomas[41], and a potential indicator for risk assessment and prognosis prediction of $\mathrm{GC}$ according to its polymorphisms [42] and bioinformatics analysis [43]. Our study found that the expression of THBS4 is notable increased following the advanced procession of GC. Moreover, THBS4 expression is associated with TGF $\beta$ in GC. TGF $\beta /$ PDGFR $\beta$ pathways might also facilitate THBS4 secretion to promoting GC. THBS4 also positively correlated with others immunoinhibitors and majority immunostimulators. The important role of THBS4 in GC needs more evidence to confirm.

CHRDL1, a paralog of CHRDL2, only two papers reported in GC. One paper reported that its overexpression correlated with poor prognosis in GC[44]. Another reported low expression of CHRDL1, as an antagonist of bone morphogenetic protein 4 (BMF4), might promote GC cell proliferation and migration by BMP receptor II [44]. However, our results revealed that high expression of CHRDL1 with poor OS and its expression associated with tumor stages, immune cell infiltration, immunoinhibitors, and immunostimulators.

Many papers showed ANGPTL1 acted as a tumor suppressor by inhibiting angiogenesis, cancer metastasis, cancer stemness, and repressing sorafenib resistance in treatment [45, 46]. while its role in GC still not clear [47]. Our results revealed that ANGPTL1 might be a potential target in GC, according to its high expression related to poor OS and gradually increased with tumor stages as well as the feasible relationship with immune cells and biomarkers in TME. 


\section{Conclusions}

Here, we searched a series of databases to find out the most relevant molecular that their expression closely related to the survival of GC. And functional enrichment analysis of survival-related CMS genes was performed. These genes are involved in many cancers, including GC, and cancer processes, such as angiogenesis. Then, we picked 6 genes that could be secreted in the TME and enhanced in immune cells. Further survival analysis demonstrated that high expression of OGN, CHRDL2, C2orf40, THBS4, CHRDL1, and ANGPTL1, respectively, was significantly associated with poor OS in GC patients. OGN and CHRDL2 were the firstly reported in GC. Moreover, the expression of these 6 genes prominently increased with the tumor pathological stage. In addition, we investigated the pathways of those hub genes by enrichment analysis. Ultimately, immune infiltration analysis displayed that those hub genes expression positively with CD8+ T Cell, M2 macrophage, most immunoinhibitors, and majority immunostimulators. These processes are closely relative to tumor growth and metastasis. Therefore, high expression of 6 hub genes alone or synergistically resulted in a poor prognosis in GC patients. More experiments are still needed to verify these findings.

\section{Abbreviations}

GC: Gastric Cancer; ICB: Immune checkpoint blockade; TME: tumor microenvironment; OS: overall survival; CMS: consensus molecular subtypes; TFs: transcription factors; SLRP: small leucine-rich proteoglycan; BMPs: bone 
morphogenic proteins; BNF-1: breast tumor novel factor 1; ECRG4: esophageal cancer-related gene-4; CSC: cancer stem cell.

\section{Acknowledgments}

This study is supported partially by the National Natural Science Foundation of China (grant numbers 82001159), the Science and Technology program of Jiangxi Provincial health commission (grant numbers 202130212), The Science and technology research project of the Education Department of Jiangxi Province (grant numbers GJJ200229), Technology Program of Jiangxi Administration of Traditional Chinese Medicine (grant numbers 2020A0247).

\section{Authors' contributions}

Mei Ma, Bin Yu, and Xin Yu performed the experimental design and data analysis. Mei Ma wrote the manuscript. Jianping Xiong supervised the study. All authors read and approved the final manuscript.

\section{Data availability}

All data generated or analyzed during this study are included in this article.

\section{Compliance with ethical standards}

Conflict of interests The authors have no conflicts of interest to disclose.

Ethics approval The study was approved by the Ethics Committee of The First Affiliated Hospital of Nanchang University.

Consent to participate Not applicable.

Consent for publication Not applicable.

Code availability Not applicable. 


\section{Materials and Methods}

\section{COMSUC}

COMSUC[12] (http://comsuc.bioinforai.tech/home) is used to identify Consensus Molecular Subtypes (CMS) by integrating multiple clustering results based on multiple platforms, multiple omics data, and multiple methods. In this study, we integrated clustering results of GC data from TCGA into two groups by three algorithms based on K-means, hierarchical clustering (Hclust), non-negative matrix factorization (NMF).

\section{Metascape}

Metascape[13] (http://metascape.org/gp/index.html\#/main/step1) is a web server designed to provide an extensive gene list annotation and analysis resource for users. Enrichment analysis was the essential part of Metascape. Here, we analyzed the prominent changed genes of CMS1 for enrichment analysis by custom analysis.

\section{Kaplan-Meier Plotter}

Kaplan-Meier plotter[18] (http://kmplot.com/analysis/) is a platform utilized to discover and validate the survival biomarkers of four cancers, including gastric cancer. To analyze the prognostic value of OGN, CHRDL2, C2orf40, THBS4, CHRDL1, and ANGPTL1, the cohorts were divided into high- and low- groups through their expression. In this study, the overall survival of 6 hub genes was compared in GC. And the hazard ratios (HRs, with 95\% confidence intervals) and log-rank P-values $(<0.05$ as significant difference) were counted.

\section{GEPIA}

Gene Expression Profiling Interactive Analysis[21] (GEPIA, http://gepia.cancer- 
pku.cn/) uses a standard processing approach to examine genes expression by RNA sequencing data for 8,587 normal samples and 9,736 tumors from GTEx and TCGA projects. Here, GEPIA was used to investigate the six hub genes expression correlation for GC tumor stages from TCGA data. In addition, we valued the correlation between the expression of CSF1R/CXCL12 and six hub genes base on GEPIA.

\section{TIMER}

TIMER[22] (https://cistrome.shinyapps.io/timer/) is a web tool used to investigate immune cell infiltration in diverse cancers, including GC. It provides data to evaluate the associations between expression levels of selected genes and infiltrating immune cells, such as B cells, CD4+ T cells, CD8+ T cells, neutrophils, macrophages, and dendritic cells. In our study, we estimated the correlation of expression of OGN, CHRDL2, C2orf40, THBS4, CHRDL1, and ANGPTL1 with immune cell infiltration.

\section{TISIDB}

TISIDB[23] (http://cis.hku.hk/TISIDB/index.php) is an online web for assessing tumor-related gene and immune system interaction. In our study, we used TISIDB to find out the correlation between the expression of six hub genes and the abundance of immunomodulators. And distribution of six hub genes expression across immune and molecular subtypes in GC. The correlations between six hub genes and the immune system were measured by Spearman's test.

\section{References}

1. Sung H, Ferlay J, Siegel RL, Laversanne M, Soerjomataram I, Jemal A, Bray F (2021) Global cancer statistics 2020: GLOBOCAN estimates of incidence and 
mortality worldwide for 36 cancers in 185 countries. CA Cancer J Clinhttps://doi.org/10.3322/caac. 21660

2. Allemani C et al. (2018) Global surveillance of trends in cancer survival 200014 (CONCORD-3): analysis of individual records for 37513025 patients diagnosed with one of 18 cancers from 322 population-based registries in 71 countries. Lancet 391, 1023-1075. https://doi.org/10.1016/S01406736(17)33326-3

3. Shitara K et al. (2018) Pembrolizumab versus paclitaxel for previously treated, advanced gastric or gastro-oesophageal junction cancer (KEYNOTE-061): a randomised, open-label, controlled, phase 3 trial. Lancet 392, 123-133. https://doi.org/10.1016/S0140-6736(18)31257-1

4. Reiser J, Banerjee A (2016) Effector, Memory, and Dysfunctional CD8(+) T Cell Fates in the Antitumor Immune Response. J Immunol Res 2016, 8941260. https://doi.org/10.1155/2016/8941260

5. Wang Y, Zhu C, Song W, Li J, Zhao G, Cao H (2018) PD-L1 Expression and CD8(+) T Cell Infiltration Predict a Favorable Prognosis in Advanced Gastric Cancer. J Immunol Res 2018, 4180517. https://doi.org/10.1155/2018/4180517

6. Shen Y, Teng Y, Lv Y, Zhao Y, Qiu Y, Chen W, Wang L, Wang Y, Mao F, Cheng P, Ma D, Zhuang Y, Zou Q, Peng L (2020) PD-1 does not mark tumor-infiltrating $\mathrm{CD} 8+\mathrm{T}$ cell dysfunction in human gastric cancer. J Immunother Cancer 8https://doi.org/10.1136/jitc-2019-000422

7. Hashimoto M, Kamphorst AO, Im SJ, Kissick HT, Pillai RN, Ramalingam SS, Araki K, Ahmed R (2018) CD8 T Cell Exhaustion in Chronic Infection and Cancer: Opportunities for Interventions. Annu Rev Med 69, 301-318. https://doi.org/10.1146/annurev-med-012017-043208

8. Wang J, Li R, Cao Y, Gu Y, Fang H, Fei Y, Lv K, He X, Lin C, Liu H, Zhang H, Li H, He H, Xu J, Huang H (2021) Intratumoral CXCR5(+)CD8(+)T associates with favorable clinical outcomes and immunogenic contexture in gastric cancer. Nat Commun 12, 3080. https://doi.org/10.1038/s41467-021-23356-w

9. Zhou Z, Xia G, Xiang Z, Liu M, Wei Z, Yan J, Chen W, Zhu J, Awasthi N, Sun X, Fung KM, He Y, Li M, Zhang C (2019) A C-X-C Chemokine Receptor Type 2-Dominated Cross-talk between Tumor Cells and Macrophages Drives Gastric Cancer Metastasis. Clin Cancer Res 25, 3317-3328. https://doi.org/10.1158/1078-0432.CCR-18-3567

10. Li Q, Wu W, Gong D, Shang R, Wang J, Yu H (2021) Propionibacterium acnes overabundance in gastric cancer promote M2 polarization of macrophages via a TLR4/PI3K/Akt signaling. Gastric Cancerhttps://doi.org/10.1007/s10120$\underline{021-01202-8}$

11. Kim KJ, Wen XY, Yang HK, Kim WH, Kang GH (2015) Prognostic Implication of M2 Macrophages Are Determined by the Proportional Balance of Tumor Associated Macrophages and Tumor Infiltrating Lymphocytes in MicrosatelliteUnstable Gastric Carcinoma. PLoS One 10, e0144192. https://doi.org/10.1371/journal.pone.0144192

12. He S, Song X, Yang X, Yu J, Wen Y, Wu L, Yan B, Feng J, Bo X (2021) 
COMSUC: A web server for the identification of consensus molecular subtypes of cancer based on multiple methods and multi-omics data. PLoS Comput Biol 17, e1008769. https://doi.org/10.1371/journal.pcbi.1008769

13. Zhou Y, Zhou B, Pache L, Chang M, Khodabakhshi AH, Tanaseichuk O, Benner C, Chanda SK (2019) Metascape provides a biologist-oriented resource for the analysis of systems-level datasets. Nat Commun 10, 1523. https://doi.org/10.1038/s41467-019-09234-6

14. Wu H, Liu X, Gong P, Song W, Zhou M, Li Y, Zhao Z, Fan H (2018) Elevated TFAP4 regulates lncRNA TRERNA1 to promote cell migration and invasion in gastric cancer. Oncol Rep 40, 923-931. https://doi.org/10.3892/or.2018.6466

15. Wadhwa R, Song S, Lee JS, Yao Y, Wei Q, Ajani JA (2013) Gastric cancermolecular and clinical dimensions. Nat Rev Clin Oncol 10, 643-55. https://doi.org/10.1038/nrclinonc.2013.170

16. Rao M, Zhu Y, Cong X, Li Q (2017) Knockdown of CREB1 inhibits tumor growth of human gastric cancer in vitro and in vivo. Oncol Rep 37, 3361-3368. https://doi.org/10.3892/or.2017.5636

17. Ashrafizadeh M, Najafi M, Ang HL, Moghadam ER, Mahabady MK, Zabolian A, Jafaripour L, Bejandi AK, Hushmandi K, Saleki H, Zarrabi A, Kumar AP (2020) PTEN, a Barrier for Proliferation and Metastasis of Gastric Cancer Cells: From Molecular Pathways to Targeting and Regulation. Biomedicines 8https://doi.org/10.3390/biomedicines8080264

18. Nagy A, Munkacsy G, Gyorffy B (2021) Pancancer survival analysis of cancer hallmark genes. Sci Rep 11, 6047. https://doi.org/10.1038/s41598-021-84787$\underline{5}$

19. Xia Y, Wang L, Xu Z, Kong R, Wang F, Yin K, Xu J, Li B, He Z, Wang L, Xu H, Zhang D, Yang L, Wu JY, Xu Z (2019) Reduced USP33 expression in gastric cancer decreases inhibitory effects of Slit2-Robol signalling on cell migration and EMT. Cell Prolif 52, e12606. https://doi.org/10.1111/cpr.12606

20. Shi R, Yang Z, Liu W, Liu B, Xu Z, Zhang Z (2014) Knockdown of Slit2 promotes growth and motility in gastric cancer cells via activation of AKT/betacatenin. Oncol Rep 31, 812-8. https://doi.org/10.3892/or.2013.2887

21. Tang Z, Li C, Kang B, Gao G, Li C, Zhang Z (2017) GEPIA: a web server for cancer and normal gene expression profiling and interactive analyses. Nucleic Acids Res 45, W98-W102. https://doi.org/10.1093/nar/gkx247

22. Li T, Fan J, Wang B, Traugh N, Chen Q, Liu JS, Li B, Liu XS (2017) TIMER: A Web Server for Comprehensive Analysis of Tumor-Infiltrating Immune Cells. Cancer Res 77, e108-e110. https://doi.org/10.1158/0008-5472.CAN-17-0307

23. Ru B, Wong CN, Tong Y, Zhong JY, Zhong SSW, Wu WC, Chu KC, Wong CY, Lau CY, Chen I, Chan NW, Zhang J (2019) TISIDB: an integrated repository portal for tumor-immune system interactions. Bioinformatics 35, 4200-4202. https://doi.org/10.1093/bioinformatics/btz210

24. Mei Y, Du Z, Hu C, Greenwald NF, Abedalthagafi M, Agar NYR, Dunn GP, Bi WL, Santagata S, Dunn IF (2017) Osteoglycin promotes meningioma development through downregulation of NF2 and activation of mTOR signaling. 
Cell Commun Signal 15, 34. https://doi.org/10.1186/s12964-017-0189-7

25. Xu T, Zhang R, Dong M, Zhang Z, Li H, Zhan C, Li X (2019) Osteoglycin (OGN) Inhibits Cell Proliferation and Invasiveness in Breast Cancer via PI3K/Akt/mTOR Signaling Pathway. Onco Targets Ther 12, 10639-10650. https://doi.org/10.2147/OTT.S222967

26. Hu X, Li YQ, Li QG, Ma YL, Peng JJ, Cai SJ (2018) Osteoglycin-induced VEGF Inhibition Enhances T Lymphocytes Infiltrating in Colorectal Cancer. EBioMedicine 34, 35-45. https://doi.org/10.1016/j.ebiom.2018.07.021

27. Hu X, Li YQ, Li QG, Ma YL, Peng JJ, Cai SJ (2018) Osteoglycin (OGN) reverses epithelial to mesenchymal transition and invasiveness in colorectal cancer via EGFR/Akt pathway. J Exp Clin Cancer Res 37, 41. https://doi.org/10.1186/s13046-018-0718-2

28. Baek SH, Cha RH, Kang SW, Park CW, Cha DR, Kim SG, Yoon SA, Kim S, Han SY, Park JH, Chang JH, Lim CS, Kim YS, Na KY (2017) Higher Serum Levels of Osteoglycin Are Associated with All-Cause Mortality and Cardiovascular and Cerebrovascular Events in Patients with Advanced Chronic Kidney Disease. Tohoku J Exp Med 242, 281-290. https://doi.org/10.1620/tjem.242.281

29. Seki T, Saita E, Kishimoto Y, Ibe S, Miyazaki Y, Miura K, Ohmori R, Ikegami Y, Kondo K, Momiyama Y (2018) Low Levels of Plasma Osteoglycin in Patients with Complex Coronary Lesions. J Atheroscler Thromb 25, 1149-1155. https://doi.org/10.5551/jat.43059

30. Sun J, Liu X, Gao H, Zhang L, Ji Q, Wang Z, Zhou L, Wang Y, Sui H, Fan Z, Li Q (2017) Overexpression of colorectal cancer oncogene CHRDL2 predicts a poor prognosis. Oncotarget 8, 11489-11506. https://doi.org/10.18632/oncotarget.14039

31. Chen H, Pan R, Li H, Zhang W, Ren C, Lu Q, Chen H, Zhang X, Nie Y (2021) CHRDL2 promotes osteosarcoma cell proliferation and metastasis through the BMP-9/PI3K/AKT pathway. Cell Biol Int 45, 623-632. https://doi.org/10.1002/cbin.11507

32. Wu I, Moses MA (2003) BNF-1, a novel gene encoding a putative extracellular matrix protein, is overexpressed in tumor tissues. Gene 311, 105-10. https://doi.org/10.1016/s0378-1119(03)00563-8

33. Wu Y, Xiang Q, Lv X, Xiang X, Feng Z, Tian S, Tang J, Xiang T, Gong J (2021) C2orf40 inhibits hepatocellular carcinoma through interaction with UBR5. J Gastroenterol Hepatolhttps://doi.org/10.1111/jgh.15441

34. Deng P, Chang XJ, Gao ZM, Xu XY, Sun AQ, Li K, Dai DQ (2018) Downregulation and DNA methylation of ECRG4 in gastric cancer. Onco Targets Ther 11, 4019-4028. https://doi.org/10.2147/OTT.S161200

35. Tang GY, Tang GJ, Yin L, Chao C, Zhou R, Ren GP, Chen JY, Zhang W (2019) ECRG4 acts as a tumor suppressor gene frequently hypermethylated in human breast cancer. Biosci Rep 39https://doi.org/10.1042/BSR20190087

36. Chen L, Tang H, Liu G, Xiao S, Liang D, Ma J, Yang Y, Luo H, Zhu Y, Xie F, Cheng X, Chi J, Wu X (2021) MicroRNA-196b promotes gastric cancer 
progression by targeting ECRG4. Anticancer Drugs 32, 127-137. https://doi.org/10.1097/CAD.0000000000000998

37. Muppala S, Xiao R, Krukovets I, Verbovetsky D, Yendamuri R, Habib N, Raman P, Plow E, Stenina-Adognravi O (2017) Thrombospondin-4 mediates TGF-beta-induced angiogenesis. Oncogene 36, 5189-5198. https://doi.org/10.1038/onc.2017.140

38. Kim MS, Choi HS, Wu M, Myung J, Kim EJ, Kim YS, Ro S, Ha SE, Bartlett A, Wei L, Ryu HS, Choi SC, Park WC, Kim KY, Lee MY (2020) Potential Role of PDGFRbeta-Associated THBS4 in Colorectal Cancer Development. Cancers (Basel) 12https://doi.org/10.3390/cancers12092533

39. Hou Y, Li H, Huo W (2020) THBS4 silencing regulates the cancer stem celllike properties in prostate cancer via blocking the PI3K/Akt pathway. Prostate 80, 753-763. https://doi.org/10.1002/pros.23989

40. Guo D, Zhang D, Ren M, Lu G, Zhang X, He S, Li Y (2020) THBS4 promotes HCC progression by regulating ITGB1 via FAK/PI3K/AKT pathway. FASEB J 34, 10668-10681. https://doi.org/10.1096/fj.202000043R

41. Forster S, Gretschel S, Jons T, Yashiro M, Kemmner W (2011) THBS4, a novel stromal molecule of diffuse-type gastric adenocarcinomas, identified by transcriptome-wide expression profiling. Mod Pathol 24, 1390-403. https://doi.org/10.1038/modpathol.2011.99

42. Lin X, Hu D, Chen G, Shi Y, Zhang H, Wang X, Guo X, Lu L, Black D, Zheng XW, Luo X (2016) Associations of THBS2 and THBS4 polymorphisms to gastric cancer in a Southeast Chinese population. Cancer Genet 209, 215-22. https://doi.org/10.1016/j.cancergen.2016.04.003

43. Lu Y, Kong X, Zhong W, Hu M, Li C (2021) Diagnostic, Therapeutic, and Prognostic Value of the Thrombospondin Family in Gastric Cancer. Front Mol Biosci 8, 647095. https://doi.org/10.3389/fmolb.2021.647095

44. Pei YF, Zhang YJ, Lei Y, Wu WD, Ma TH, Liu XQ (2017) Hypermethylation of the CHRDL1 promoter induces proliferation and metastasis by activating Akt and Erk in gastric cancer. Oncotarget 8, 23155-23166. https://doi.org/10.18632/oncotarget.15513

45. Yan Q, Jiang L, Liu M, Yu D, Zhang Y, Li Y, Fang S, Li Y, Zhu YH, Yuan YF, Guan XY (2017) ANGPTL1 Interacts with Integrin alphalbeta1 to Suppress HCC Angiogenesis and Metastasis by Inhibiting JAK2/STAT3 Signaling. Cancer Res 77, 5831-5845. https://doi.org/10.1158/0008-5472.CAN-17-0579

46. Chen HA, Kuo TC, Tseng CF, Ma JT, Yang ST, Yen CJ, Yang CY, Sung SY, Su JL (2016) Angiopoietin-like protein 1 antagonizes MET receptor activity to repress sorafenib resistance and cancer stemness in hepatocellular carcinoma. Hepatology 64, 1637-1651. https://doi.org/10.1002/hep.28773

47. Tang C, Chen E, Peng K, Wang H, Cheng X, Wang Y, Yu S, Yu Y, Cui Y, Liu T (2020) Mining the role of angiopoietin-like protein family in gastric cancer and seeking potential therapeutic targets by integrative bioinformatics analysis. Cancer Med 9, 4850-4863. https://doi.org/10.1002/cam4.3100 
Figures and Figure legends

a
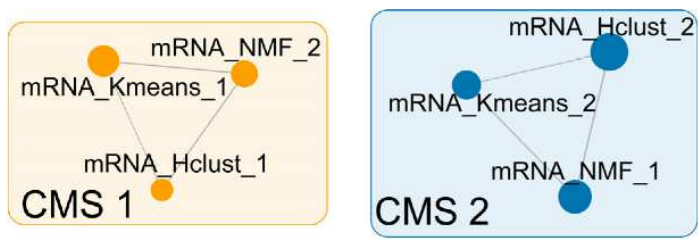

b

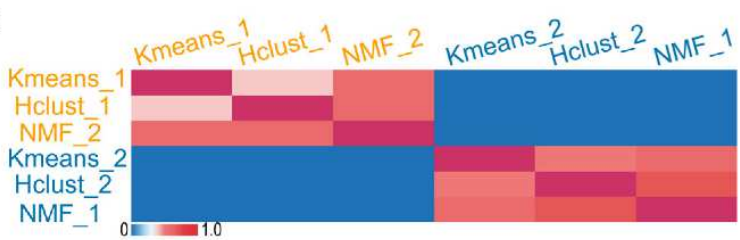

d

CMS 1

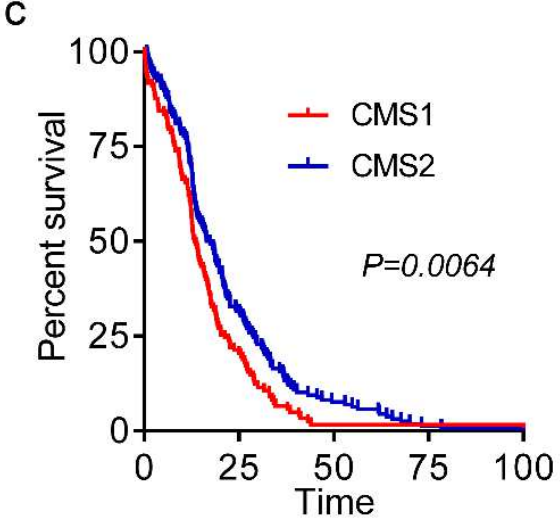

CMS 2

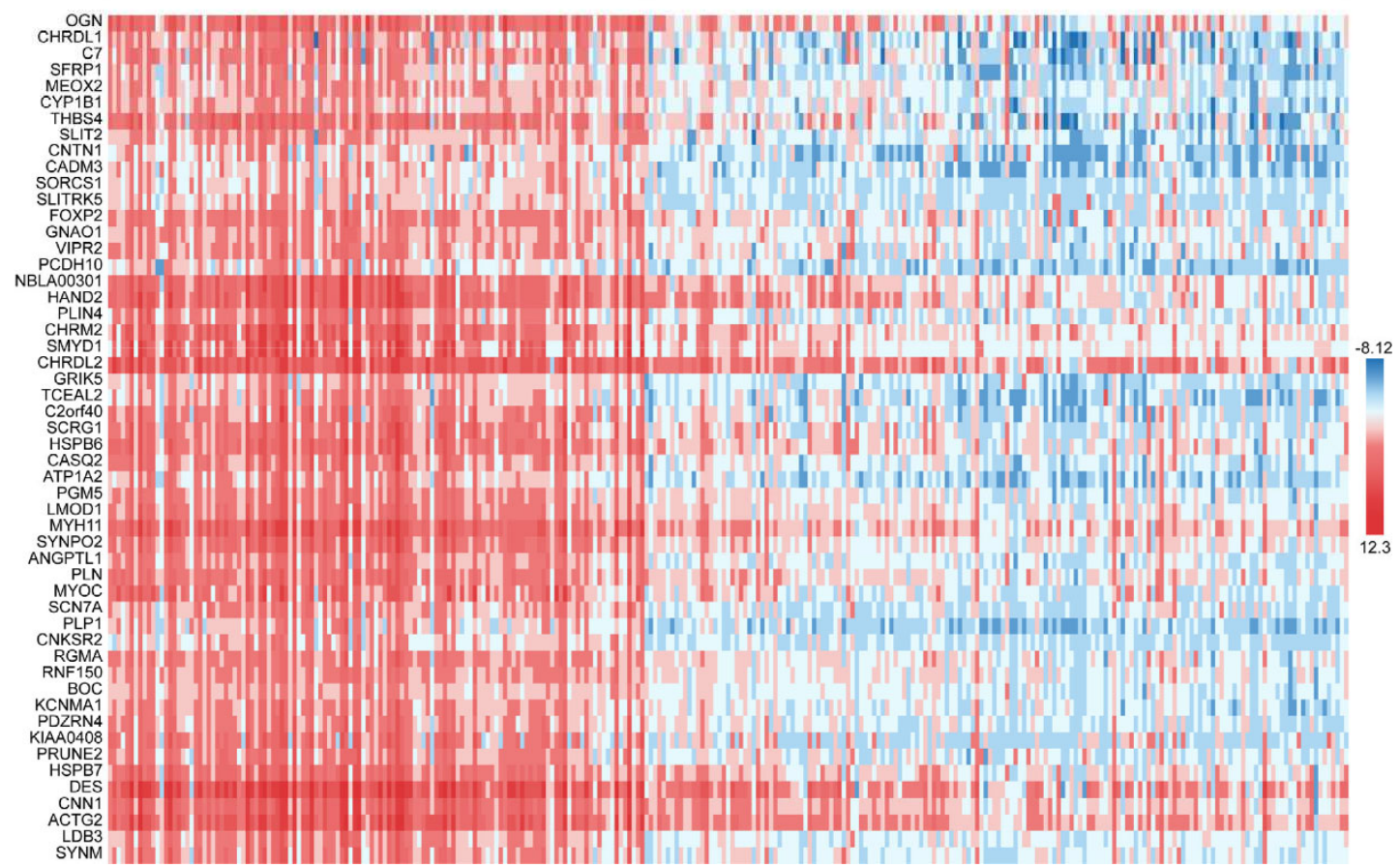

Fig. 1 Identification of robust genes associated with GC survival.

a Network of GC CMS by K-means, hierarchical clustering (Hclust), non-negative matrix factorization (NMF). b Heatmap of GC by two groups of CMS across three clustering methods. c Kaplan-Meier survival analysis showed CMS1 had poor survival. d Identification of key modules that prominent change in CMS1 vs. CMS2. 
a
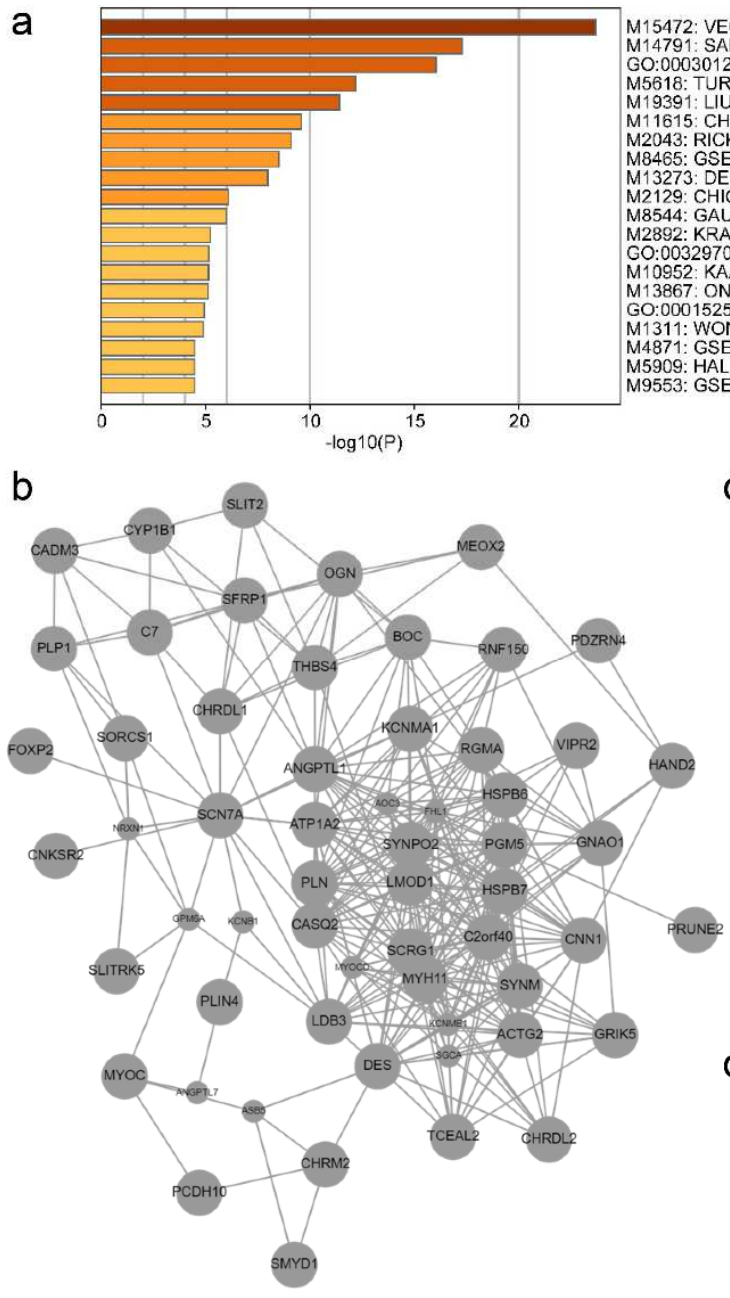

C

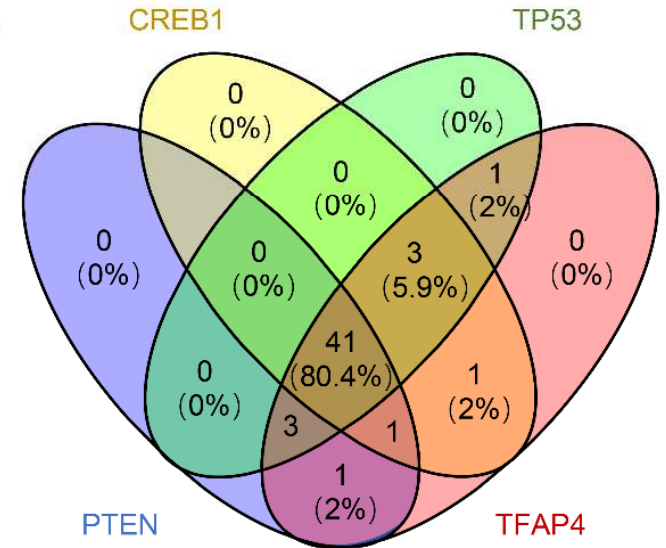

d

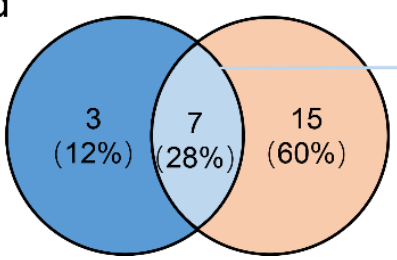

OGN, CHRDL2,

C2orf40, THBS4,

CHRDL1,

ANGPTL1

Fig. 2 Functional enrichment analysis of survival-related CMS genes.

a Gene ontology (GO) enrichment analysis of CMS selected genes in Metascape. b PPI network of CMS selected genes in GENEMANIA database. $\mathbf{c}$ Venn diagram showed the number of those selected 52 genes regulated by TFAP4, TP53, CREB1, PTEN predicted in Knock TF. d Venn diagram displayed genes that encode secreted proteins and enriched in immune cells. 
a

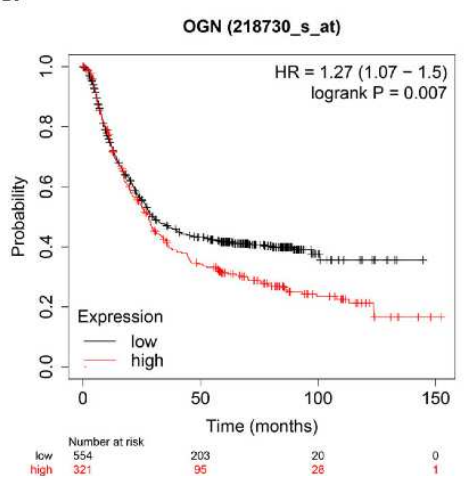

d

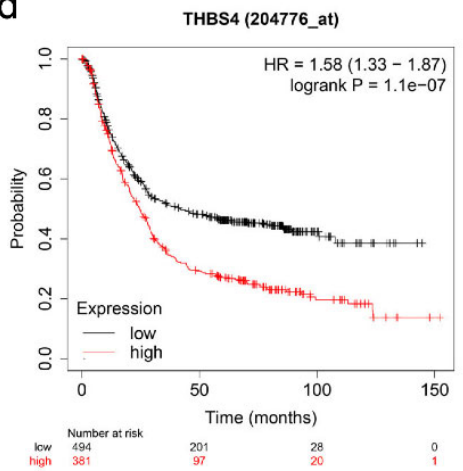

b

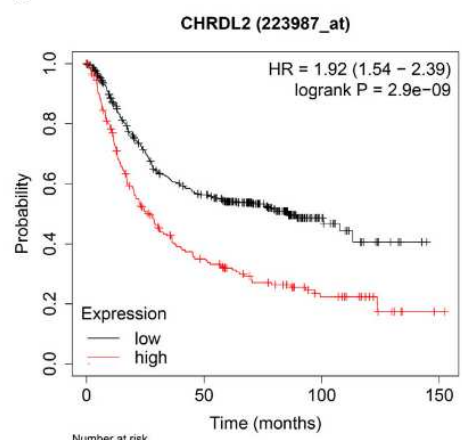

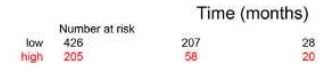

e

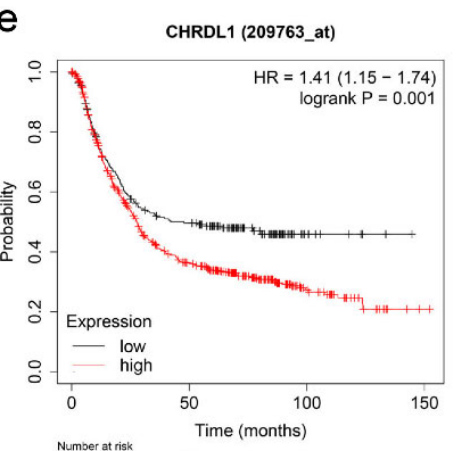

lowi
high
high
626
C

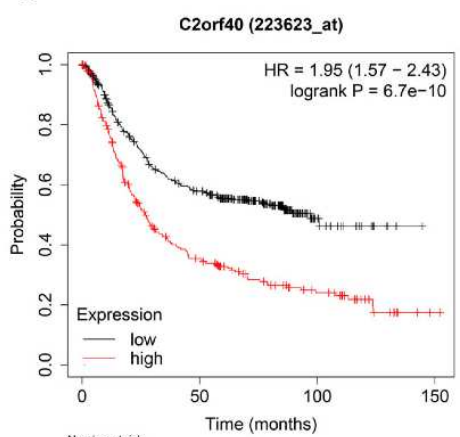

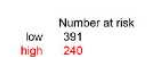

f 197
68

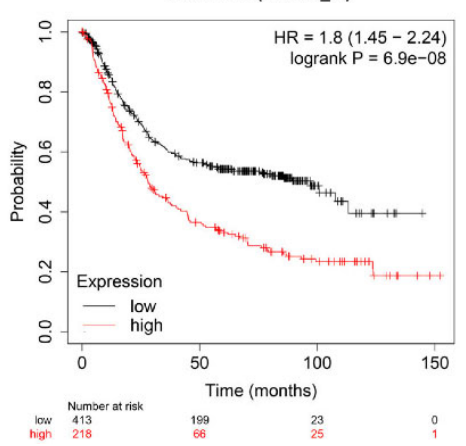

Fig. 3 Overall survival analysis of six hub genes in GC patients.

Survival curves for high and low expression of OGN, CHRDL2, C2orf40, THBS4, CHRDL1, and ANGPTL1 in GC based on the Kaplan-Meier plotter database. 

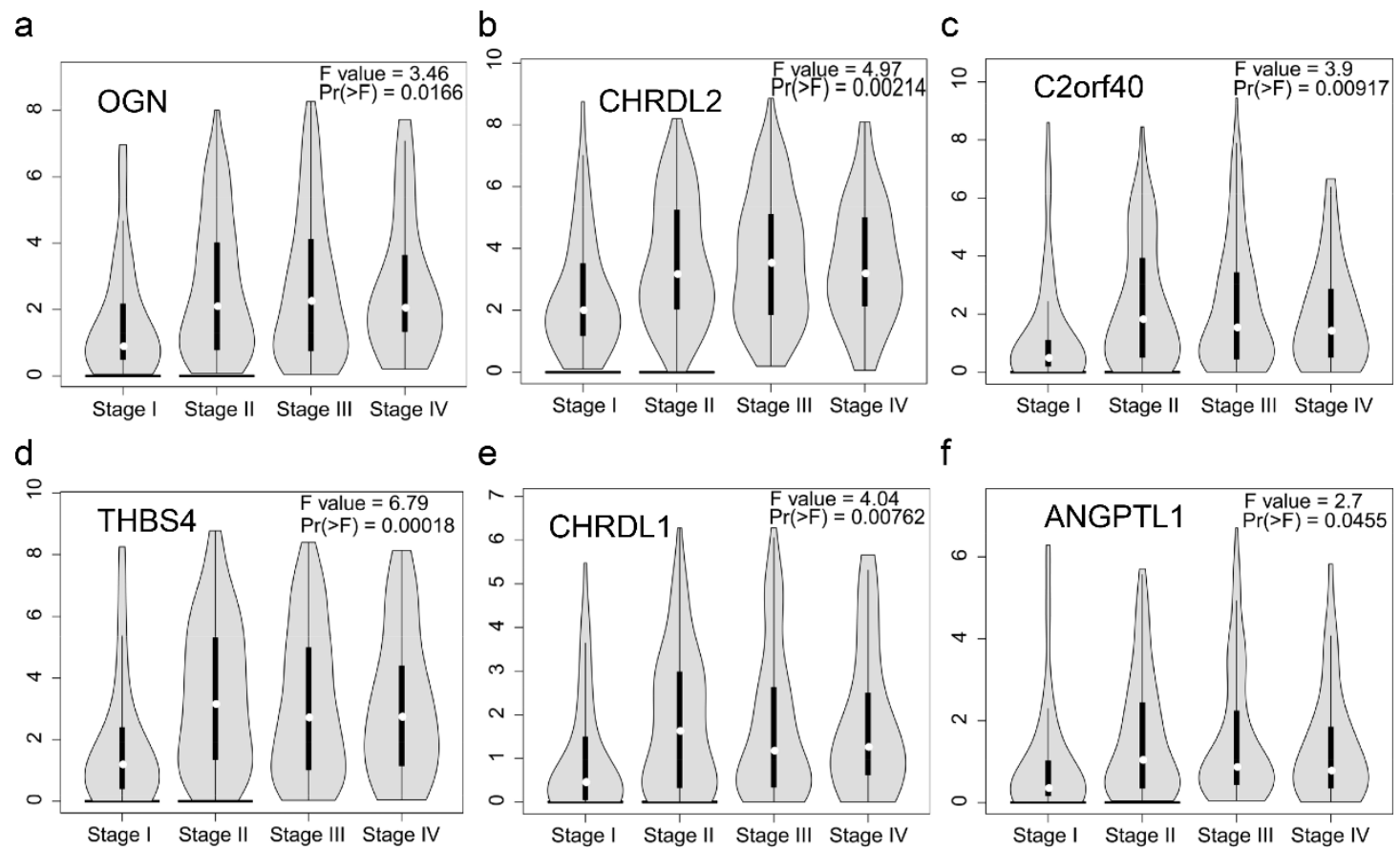

Fig. 4 Relationships between mRNA levels of six hub genes and tumor stages.

mRNA expression of OGN, CHRDL2, C2orf40, THBS4, CHRDL1, and ANGPTL1 was significantly correlated with tumor stages based on GEPIA. 

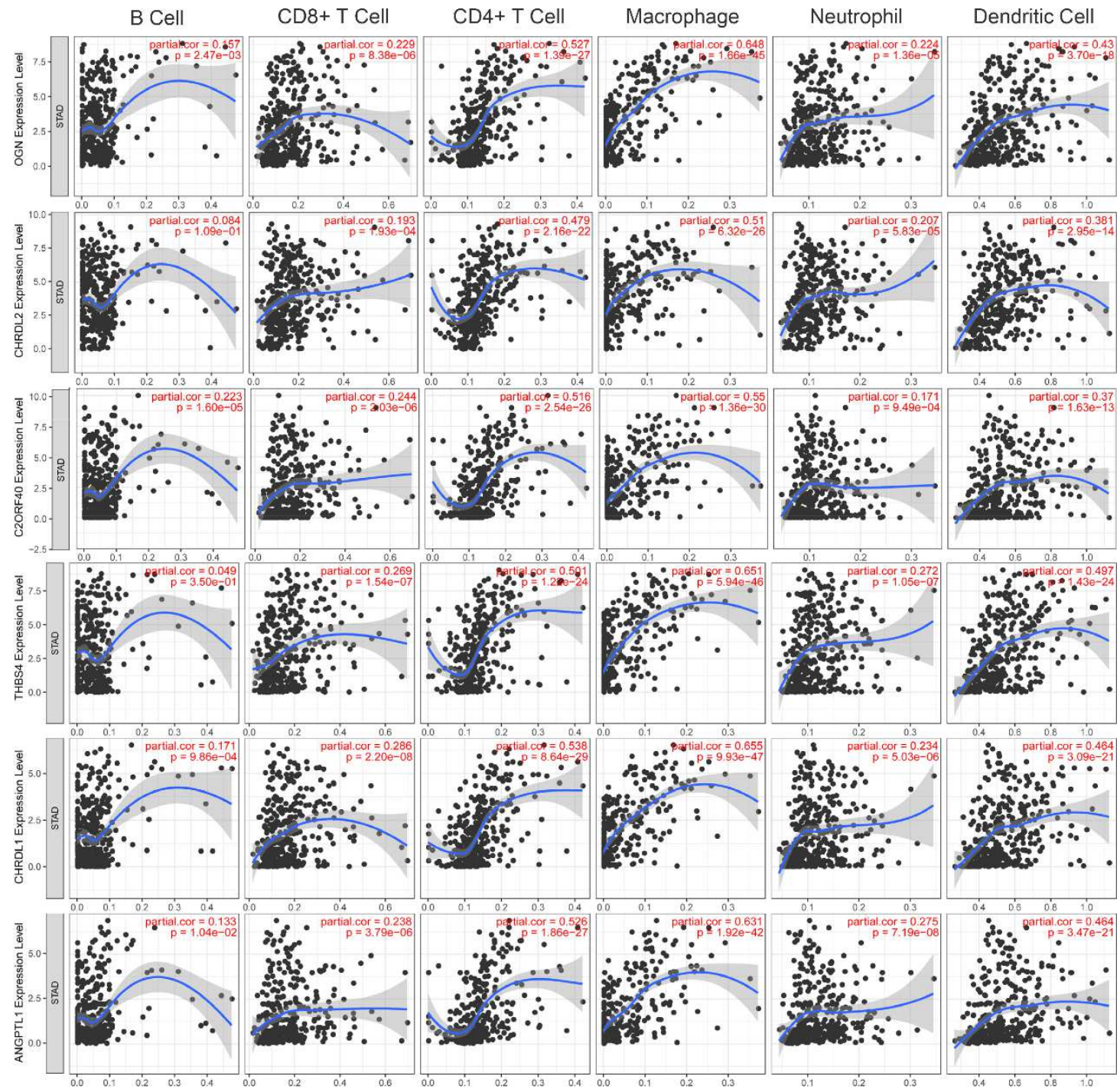

Fig.5 Correlations of six hub genes with immune cell infiltration level in GC. OGN,

CHRDL2, C2orf40, THBS4, CHRDL1, and ANGPTL1 all had significant positive correlations with infiltrating levels of CD8+ T Cell, CD4+ T Cell, macrophage, neutrophil, dendritic cell in GC. 

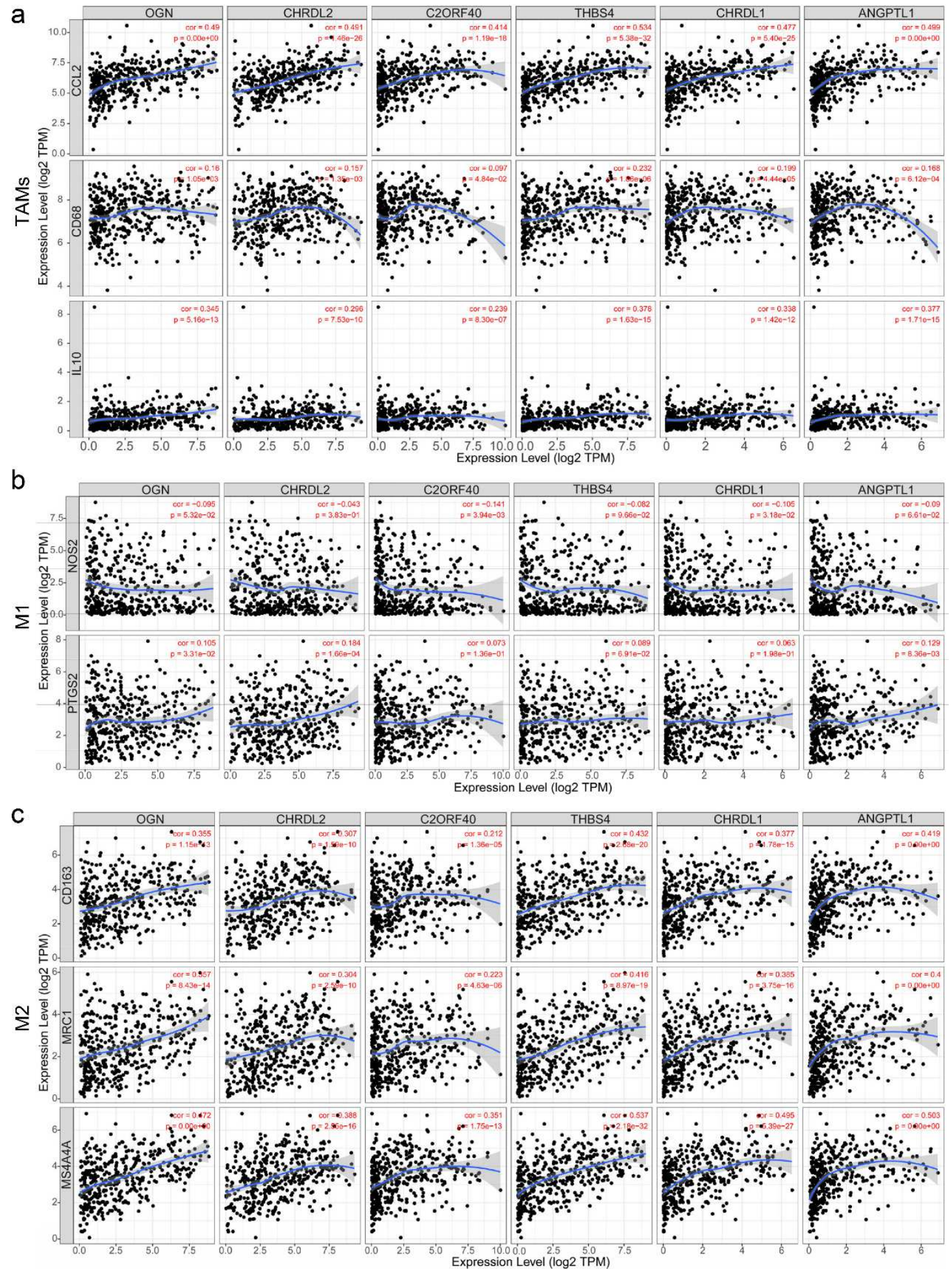

Fig. 6 Expression of six hub genes correlated with TAM polarization in the TME

of GC. Scatterplots outline the relationship between the expression of six hub genes and various gene markers of TAMs (a), M1 macrophages (b), and M2 macrophages (c) by TIMER. 

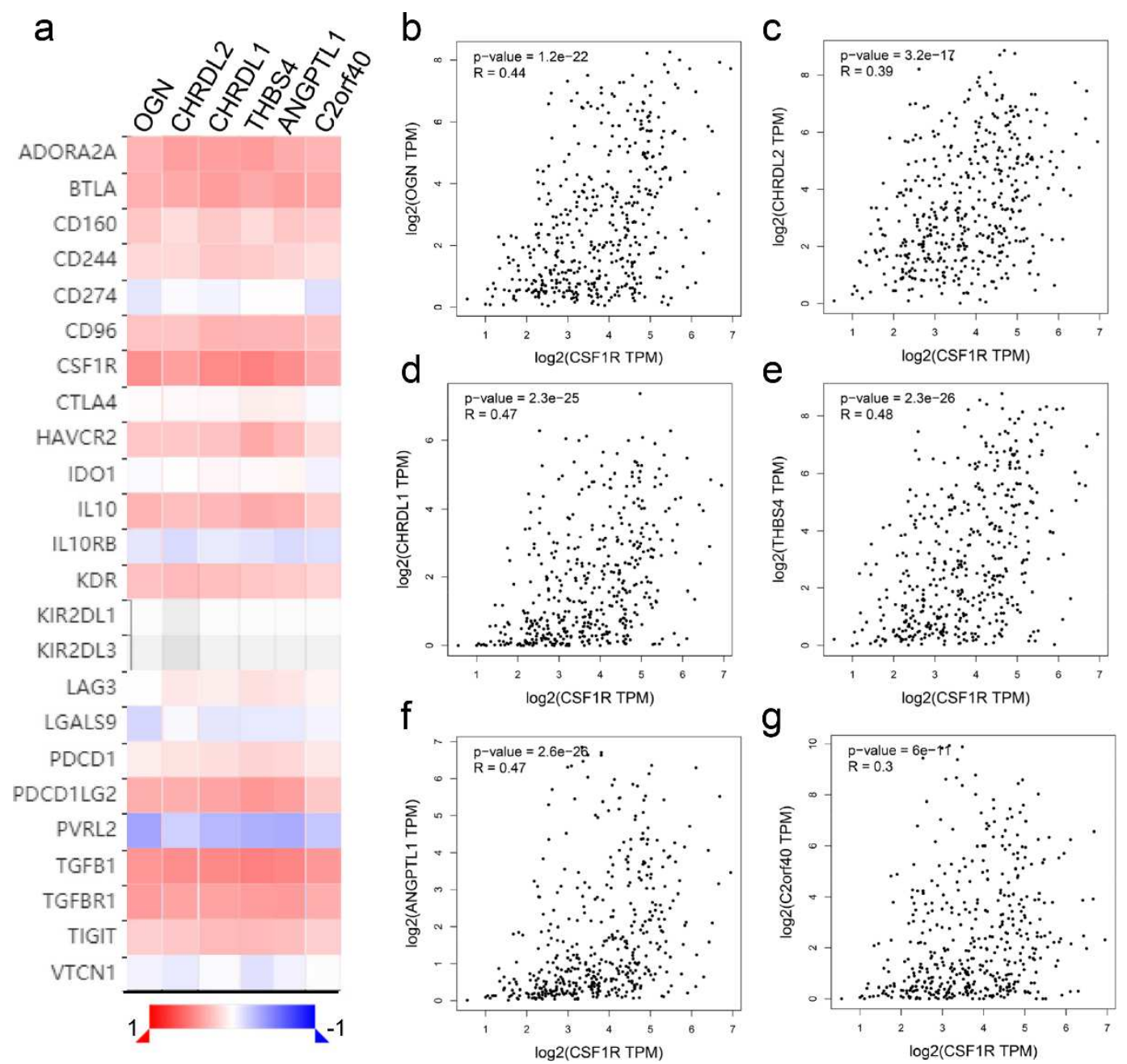

Fig. 7 Relations between the abundance of tumor-infiltrating immunoinhibitors and expression of six hub genes.

a Heatmap exhibited six hub genes that were positively correlated with most immunoinhibitors base on TISIDB. b CSF1R had linked with six hub genes base on GEPIA. 

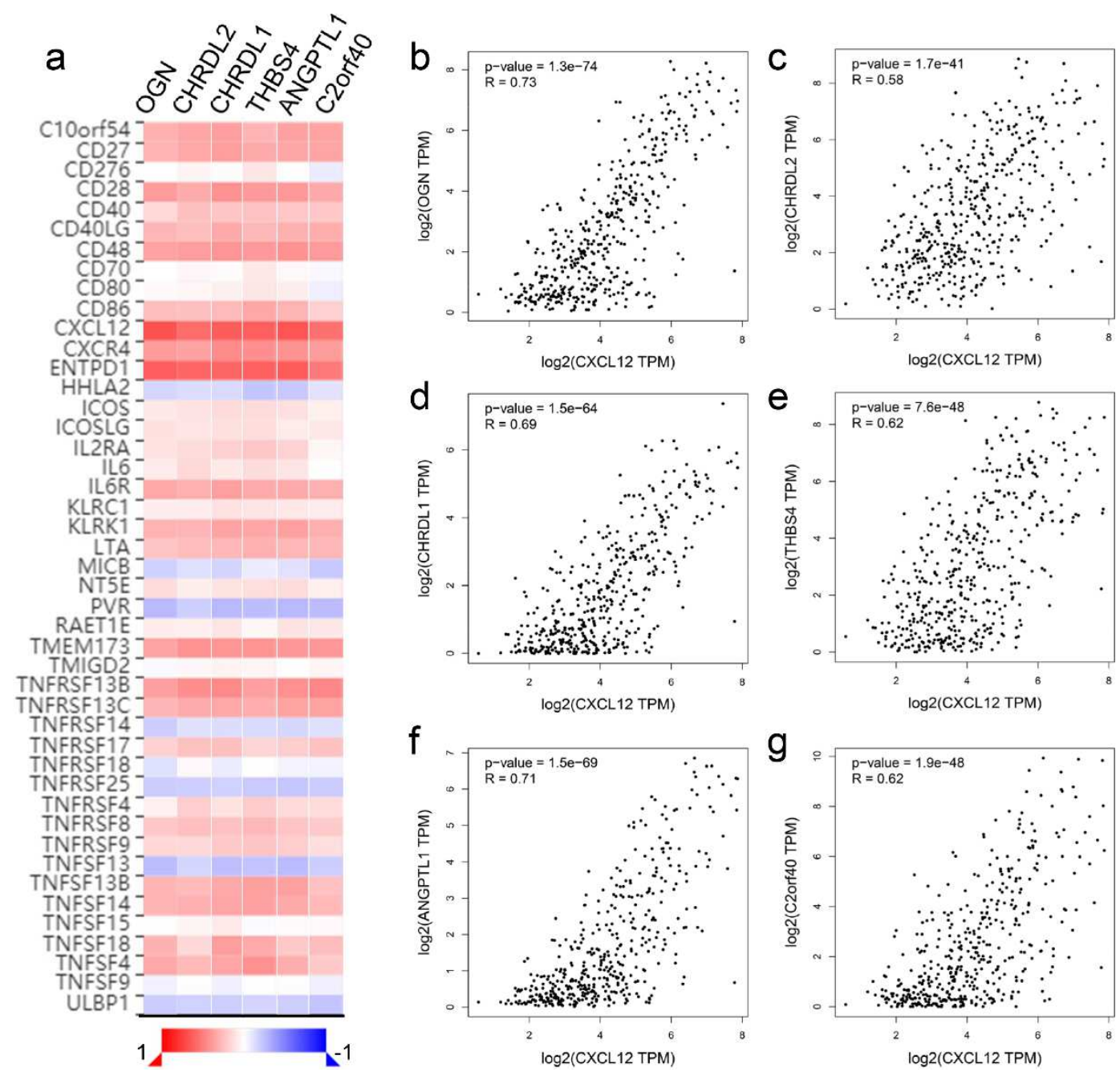

Fig. 8 Tumor immunology analysis exhibited the expression of six hub genes' relationship with immunostimulators base on TISIDB (a) and GEPIA (b-g). 

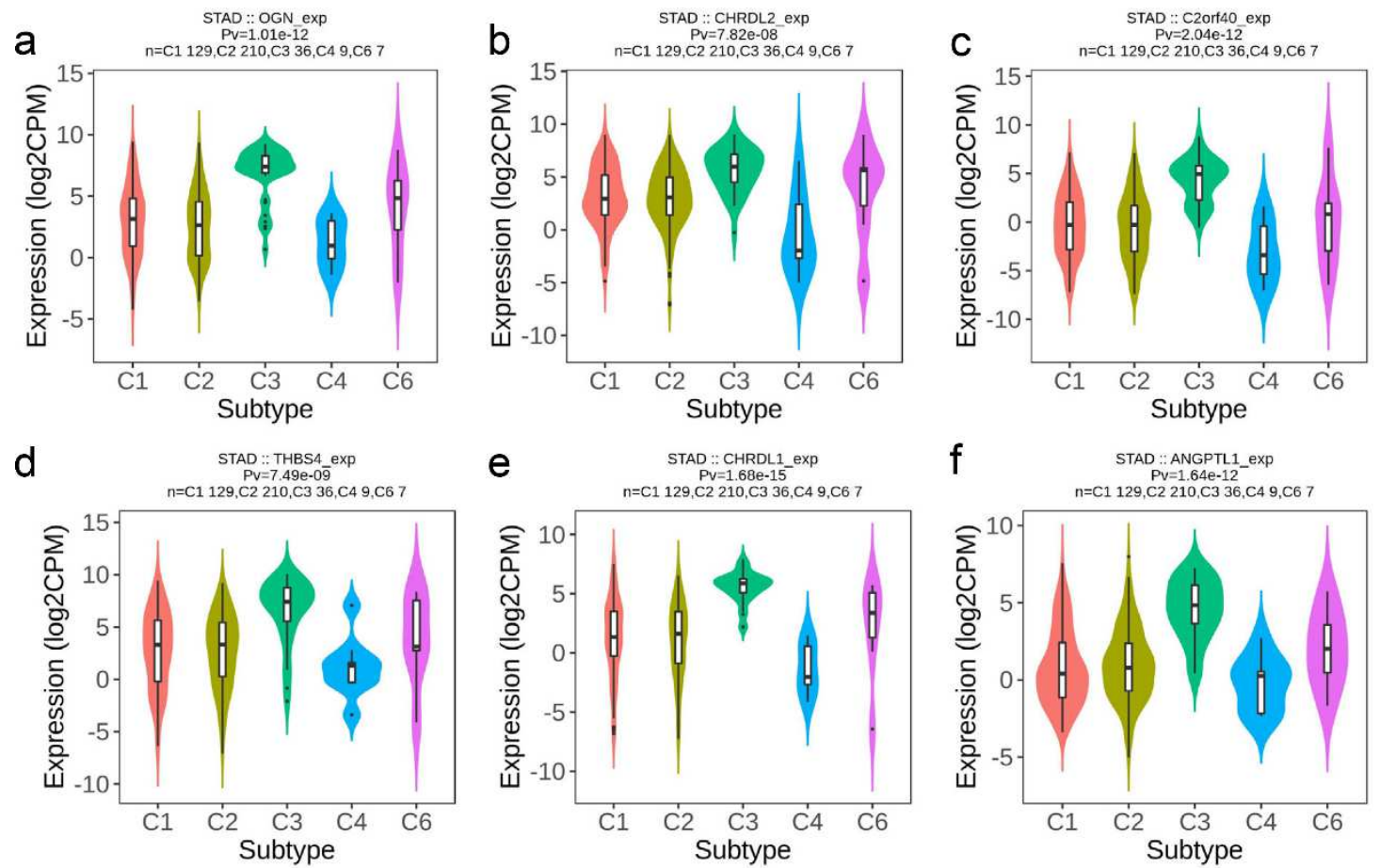

Fig. 9 Associations between expression of six hub genes and immune subtypes in

\section{GC.}

OGN (a), CHRDL2 (b), C2orf40 (c), THBS4 (d), CHRDL1(e) and ANGPTL1 (f) investigated in TISIDB. $\mathrm{C} 1$ : wound healing; $\mathrm{C} 2$ : IFN- $\gamma$ dominant; $\mathrm{C} 3$ : inflammatory; C4: lymphocyte depleted; C5: immunologically quiet; C6: TGF- $\beta$ dominant. 

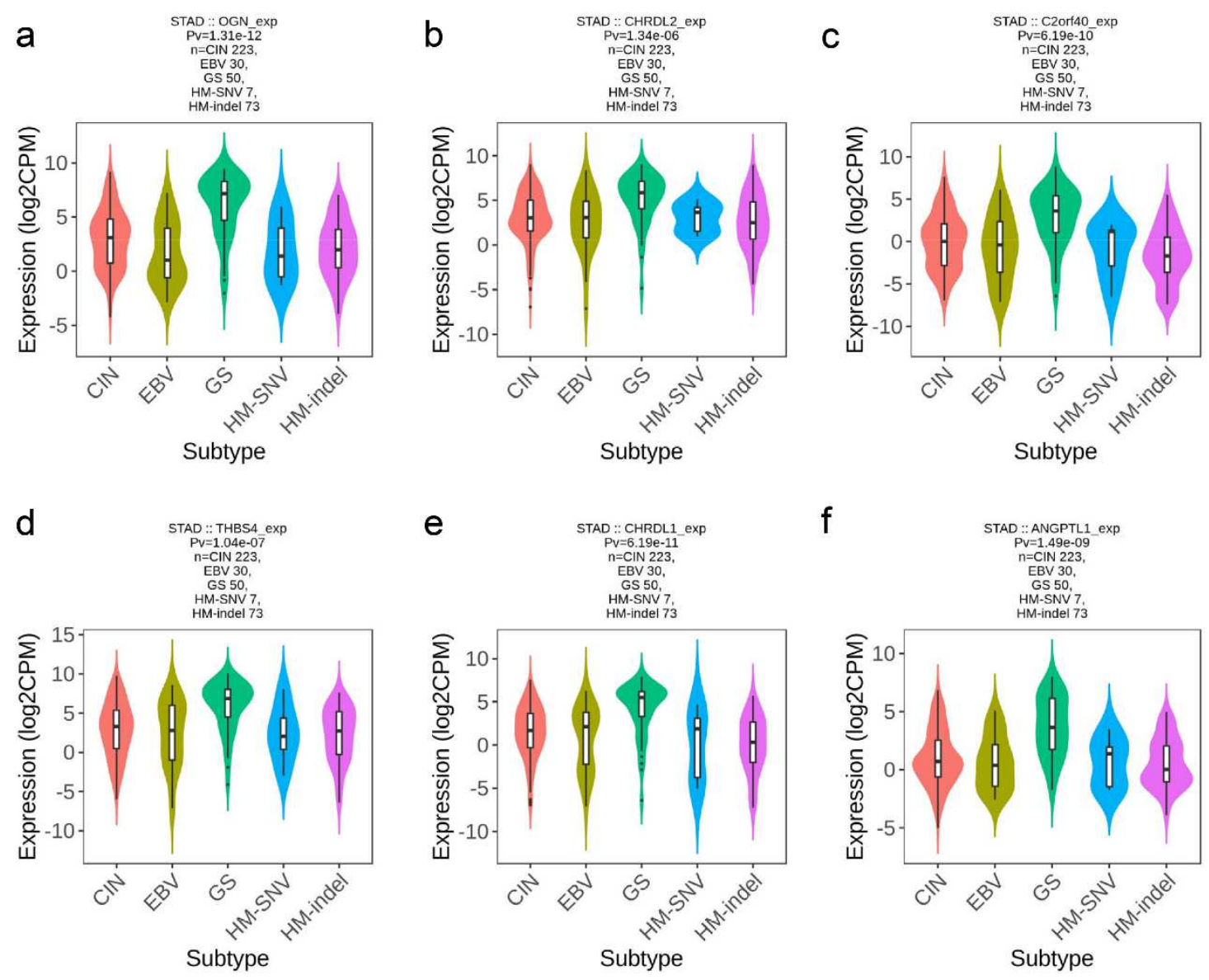

Fig. 10 Correlations between expression of six hub genes and molecular subtypes in GC.

OGN (a), CHRDL2 (b), C2orf40 (c), THBS4 (d), CHRDL1(e) and ANGPTL1 (f) analyzed in TISIDB. CIN: chromosomal instable; EBV: Epstein-Barr virus-positive; GS: genomically stable; HM-SNV: hypermutated with elevated single nucleotide variation; HM-indel: hypermutated enriched for insertion/deletion.

\section{Tables}

Table 1. Selected 52 genes with CMS1-related significant change performed TF enrichment in Knock TF.

TFs Genes regulated by relative TFs




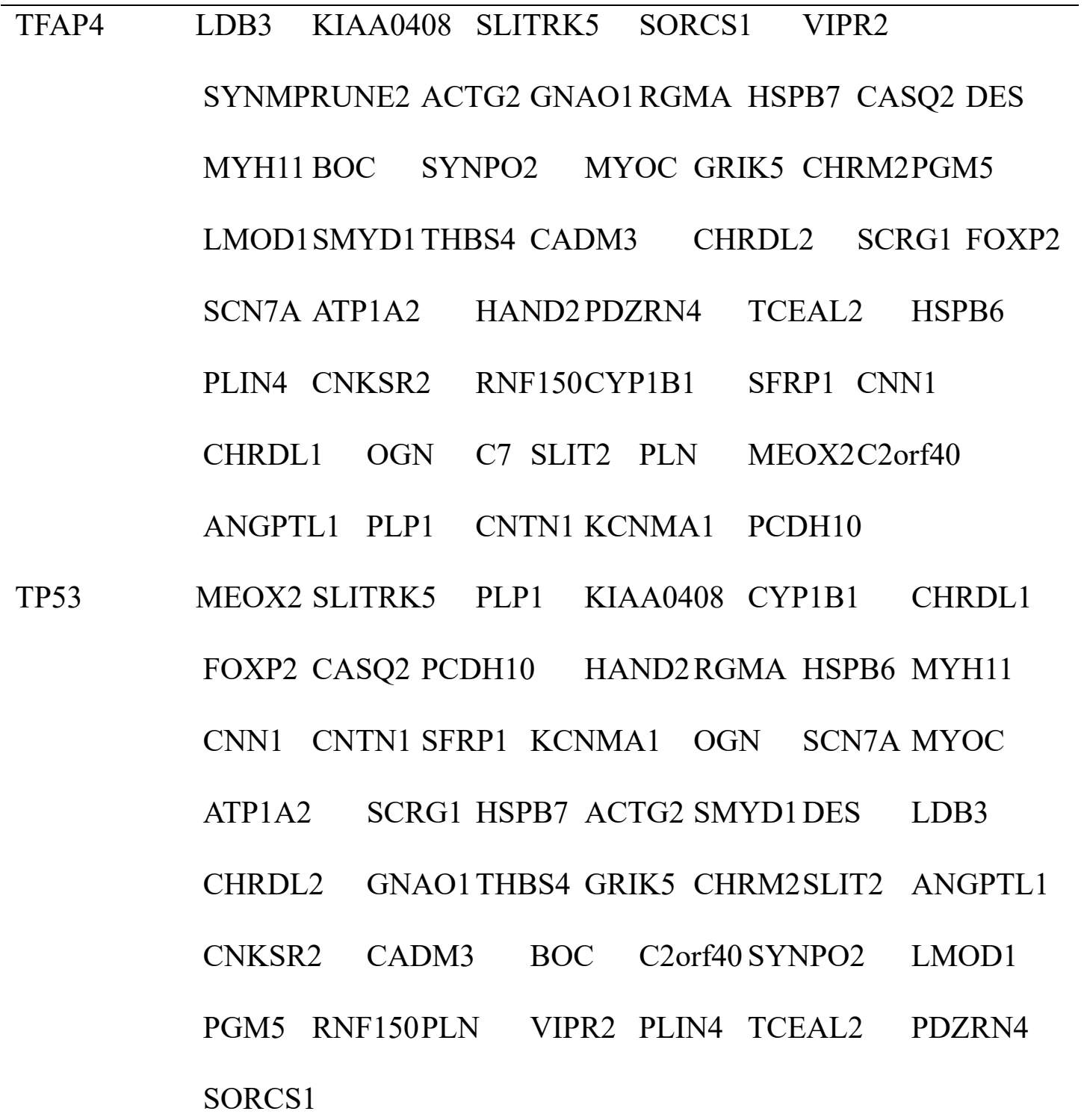

CREB1 CASQ2 SMYD1CHRM2PGM5 TCEAL2 MEOX2PLP1 C7 KIAA0408 SYNPO2 DES KCNMA1 LDB3 PLIN4 MYOC GNAO1 SCRG1 FOXP2 SLIT2 SCN7A CYP1B1 ANGPTL1 PCDH10 ATP1A2 PRUNE2 SLITRK5 CNN1 CNTN1 ACTG2 LMOD1C2orf40 PLN CADM3 SORCS1 OGN THBS4 CHRDL2 RGMA HSPB7 SFRP1 CHRDL1 GRIK5 HAND2 HSPB6 PDZRN4 CNKSR2 PTEN KIAA0408 PCDH10 CHRDL1 GNAO1CNTN1 SLITRK5 SLIT2 SMYD1SYNM SCRG1 BOC GRIK5 HSPB6 


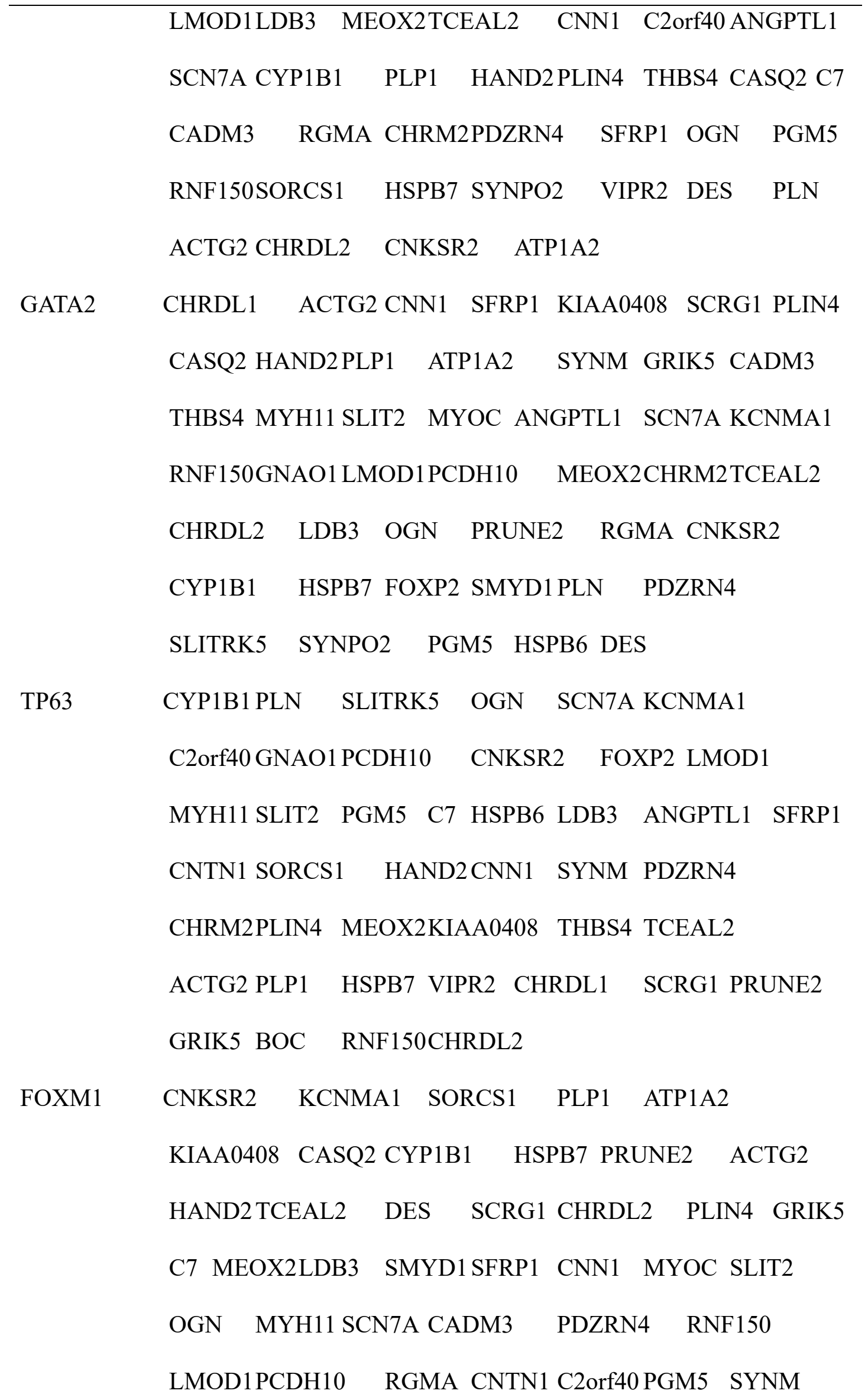


BOC HSPB6 ANGPTL1

FOXP1 CYP1B1CNN1 SORCS1 PLN LMOD1HSPB6 CHRDL2

C2orf40 MEOX2TCEAL2 PGM5 PDZRN4 ANGPTL1

CHRM2RGMA RNF150DES PLP1 HSPB7 SCN7A C7

PLIN4 VIPR2 CNKSR2 CHRDL1 CASQ2 HAND2

SLITRK5 SFRP1 OGN CADM3 PRUNE2 CNTN1

KIAA0408 MYOC LDB3 SYNM KCNMA1 SLIT2 GRIK5

GNAO1 SYNPO2

NR2F2 HSPB7 GNAO1DES KCNMA1 TCEAL2 CNTN1

SORCS1 CHRDL2 CYP1B1 SCRG1 SMYD1PLP1

SFRP1 MYH11 LDB3 CNN1 C2orf40 CHRM2ACTG2 BOC

PRUNE2 PCDH10 SLIT2 CADM3 MYOC OGN

RGMA THBS4 PDZRN4 CNKSR2 PLIN4 HAND2

KIAA0408 CHRDL1 GRIK5 SLITRK5 MEOX2C7 PGM5

SYNM ATP1A2

POSTN KIAA0408 ANGPTL1 CNN1 CHRM2C7 CASQ2 FOXP2

OGN ATP1A2 GNAO1 KCNMA1 SYNPO2 THBS4

HAND2CADM3 LMOD1CHRDL1 PLP1 ACTG2 DES

CNTN1 VIPR2 GRIK5 C2orf40 SCN7A RGMA SLIT2

RNF150HSPB7 SFRP1 CNKSR2 LDB3 MYH11 PLN

SLITRK5 CHRDL2 MEOX2SYNM 
Table 2. Characteristics of the 52 genes that can be transcribed into secreted proteins or enriched in immune cells.

Characters Selected genes

\begin{tabular}{ll}
\hline Secreted & C7 THBS4 CHRDL1 SFRP1 SLIT2 CHRDL2 SCRG1 OGN \\
& C2orf40 ANGPTL1 \\
Immune cell- & OGN CHRDL1 PLP1 VIPR2 CHRDL2 PGM5 BOC SORCS1 \\
enriched & THBS4 GRIK5 CASQ2 PLIN4 TCEAL2 C2orf40 PLN ANGPTL1 \\
& SLIT2 GNAO1 SCN7A NBLA00301 MEOX2 CHRM2 CNKSR2
\end{tabular}

